\title{
Two-step phase demodulation algorithm based on quadratic phase parameter estimation using state space analysis
}

\author{
Rishikesh Kulkarni* \\ Department of Electronics and Electrical Engineering, Indian Institute of Technology Guwahati, India. \\ Pramod Rastogi \\ Applied Computing and Mechanics Laboratory, Ecole Polytechnique Fédérale de Lausanne, 1015 \\ Lausanne, Switzerland.
}

\begin{abstract}
We propose a noise robust two-step phase shifting algorithm for the evaluation of random and unknown phase step. In this algorithm, the phase within a small size twodimensional window around a user selected pixel in an interferogram is approximated as a quadratic surface. The problem of quadratic phase fitting parameter estimation is formulated as a state space analysis performed using the extended Kalman filter. The phase step is evaluated from the estimated parameters and subsequently the interferogram phase distribution is obtained. Simulation and experimental results are demonstrated.
\end{abstract}

Key words: Phase estimation; Two-step phase shifting interferometry; Quadratic phase; State space analysis.

\section{Introdution}

Phase shifting interferometry (PSI) is one of the most commonly used techniques in the optical interferometic measurement systems wherein multiple phase shifted interferograms are recorded carrying the information on the measurand [1, 2, 3, 4, 5].

5 The important advantages that the PSI offers are high accuracy in the phase estimation, higher noise robustness and measurement with higher spatial resolution compared to

\footnotetext{
${ }^{*}$ Corresponding author
} 
the single-frame spatial interferogram analysis techniques. Numerous PSI algorithms have been reported in the literature for the purpose of phase estimation from a stack of interferograms. The important limitation associated with PSI is the requirement of its isolation from mechanical disturbances. These disturbances may bring undesirable changes in the interferograms which in turn adversely affect the accuracy of phase estimation. It is therefore preferable to obtain the phase estimate from a minimum number of interferograms which generally happens to be three. Further increase in the number of interferograms provides robustness against noise and phase step miscalibration at the cost of an increased susceptibility to vibrations.

Recently, a lot of research effort has been directed towards obtaining the phase estimate from the recording of only two phase shifted interferograms $[6,7,8,9,10,11,12$, 13, 14, 15, 16, 17, 18, 19, 20, 21, 22]. The main objective of these techniques is to do away with the effects of air turbulence, temperature variations and mechanical vibrations in PSI systems while maintaining the features offered by PSI intact. Usually, the phase estimation procedure involves two steps: phase step evaluation and interferogram demodulation. A single phase step applied between the two interferograms is generally unknown and can lie anywhere within $(0, \pi)$. Therefore, an accurate evaluation of phase step is key for the successful demodulation of interferograms. Kries et al.[6] first proposed a Fourier transform based method for the pointwise demodulation of interferograms by estimating the phase step. Another PSI algorithm for the phase step estimation based on a self-tuning (ST) quadrature filter is proposed in [7]. A two-step procedure described in [8] first evaluates the fringe direction map using a regularized optical flow (OF) algorithm. Subsequently, the spiral phase transform is applied to one of the interferograms and utilizing the estimated fringe direction map, the associated quadrature interferogram is obtained. The algorithm based on Gram-Schmidth (GS) orthonormalization method [9] computes orthonormalized interferogram basis. The phase step estimation based on the extreme values of interference (EVI) [10] obtained from one of the interferogram is found to be computationally efficient. Some of the other two-step PSI algorithms include methods based on two-dimensional continuous wavelet transform [11], quotient of the inner product [14], independent component analysis [17] and polynomial phase fitting and global optimization [19, 21]. 
In this paper, we propose an algorithm for the estimation of the phase step from two interferograms recorded in a PSI setup. The phase within a two-dimensional window around a selected pixel is approximated to be a two-dimensional quadratic function of the spatial coordinates. The quadratic phase parameters are evaluated using a state space analysis approach. The phase step estimate which is derived from the estimated phase parameters is subsequently employed to demodulate the interferogram.

\section{Theory}

Two phase shifted interferograms can be represented as

$$
\begin{aligned}
& I_{1}(x, y)=a(x, y)+b(x, y) \cos [\phi(x, y)], \\
& I_{2}(x, y)=a(x, y)+b(x, y) \cos [\phi(x, y)+\alpha],
\end{aligned}
$$

where, $a(x, y), b(x, y)$ and $\phi(x, y)$ represent background intensity, fringe amplitude and phase, respectively. $x \in[1, N]$ and $y \in[1, M]$ represent the spatial coordinates. The interferogram $I_{2}$ is phase shifted by a phase step of $\alpha$ with respect to $I_{1}$. In order to reduce the number of unknown variables, the slowly (spatially) varying background intensity can be removed by the application of high pass filtering [23]. Such high pass filtered interferograms can be represented as

$$
\begin{aligned}
& \tilde{I}_{1}(x, y)=b(x, y) \cos [\phi(x, y)], \\
& \tilde{I}_{2}(x, y)=b(x, y) \cos [\phi(x, y)+\alpha] .
\end{aligned}
$$

In the proposed method, a quadratic phase approximation is considered within a small size window around a selected pixel, say, $\left(x_{p t}, y_{p t}\right)$. The interferograms within the window can be expressed as

$$
\begin{gathered}
\tilde{I}_{1 L}(\bar{x}, \bar{y})=b \cos \left[\phi_{1}(\bar{x}, \bar{y})\right], \\
\tilde{I}_{2 L}(\bar{x}, \bar{y})=b \cos \left[\phi_{2}(\bar{x}, \bar{y})\right],
\end{gathered}
$$

where, $\bar{x}, \bar{y} \in[-L, L]$ with $x_{p t} \widehat{=}(\bar{x}(0)=0)$ and $y_{p t} \widehat{=}(\bar{y}(0)=0)$. $L$ is the window size parameter. Note that the fringe amplitude is considered to be constant within the 
analysis window. Two-dimensional quadratic phase approximation is considered as

$$
\begin{aligned}
& \phi_{1}(\bar{x}, \bar{y})=c_{0}+c_{1} \bar{x}+c_{2} \bar{y}+c_{3} \bar{x}^{2}+c_{4} \overline{x y}+c_{5} \bar{y}^{2}, \\
& \phi_{2}(\bar{x}, \bar{y})=c_{6}+c_{1} \bar{x}+c_{2} \bar{y}+c_{3} \bar{x}^{2}+c_{4} \overline{x y}+c_{5} \bar{y}^{2},
\end{aligned}
$$

where, $\alpha=c_{6}-c_{0}$. The proposed method aims to accurately estimate the quadratic phase parameters from which the phase step can be computed.

\subsection{Phase Parameter Estimation Via State Space Analysis}

The problem of parameter estimation is considered from the point of view of a state space analysis wherein the phase parameters are defined to be the elements of a state vector. The state estimation is performed using a nonlinear Kalman filter utilizing the fringe sample observations. Such an observation vector is defined as

$$
\boldsymbol{I}_{l}=\left[\begin{array}{c}
\tilde{I}_{1 L}(\bar{x}, \bar{y}) \\
\tilde{I}_{2 L}(\bar{x}, \bar{y})
\end{array}\right] .
$$

Considering a raster scanning of fringe samples within the analysis window, the variable $l$ takes the values in the range $\left[1,(2 L+1)^{2}\right]$. Now, let us define the state vector as

$$
\boldsymbol{\beta}_{l}=\left[c_{0}, c_{1}, c_{2}, c_{3}, c_{4}, c_{5}, c_{6}, b\right]^{T} .
$$

The superscript $T$ indicates the transpose operation. The process update of state vector can be represented as

$$
\boldsymbol{\beta}_{l}=\boldsymbol{F} \boldsymbol{\beta}_{l-1},
$$

where, $\boldsymbol{F}$ represents the state transition matrix. Since the phase parameters are defined to be constant within the window, the state vector is essentially a constant vector. Accordingly, $\boldsymbol{F}$ is defined to be an identity matrix of size $8 \times 8$. Based on the quadratic phase approximation, the observation vector in Eq. (9) can be modelled as

$$
\begin{aligned}
\boldsymbol{I}_{l} & =\boldsymbol{h}_{l}\left(\boldsymbol{\beta}_{l}\right), \\
& =\left[\begin{array}{l}
\boldsymbol{\beta}_{l}(8) \cos \left(\boldsymbol{\beta}_{l}^{T} \boldsymbol{p}_{1}\right) \\
\boldsymbol{\beta}_{l}(8) \cos \left(\boldsymbol{\beta}_{l}^{T} \boldsymbol{p}_{2}\right)
\end{array}\right],
\end{aligned}
$$


where,

$$
\begin{aligned}
& p_{1}=\left[1, \bar{x}, \bar{y}, \bar{x}^{2}, \overline{x y}, \bar{y}^{2}, 0,0\right]^{T}, \\
& p_{2}=\left[0, \bar{x}, \bar{y}, \bar{x}^{2}, \overline{x y}, \bar{y}^{2}, 1,0\right]^{T} .
\end{aligned}
$$

Since the observation is a nonlinear function of state vector (See Eq. (13)), we propose to use the extended Kalman filter (EKF) for the estimation of phase parameters.

The procedure of EKF based parameter estimation is described as follows [24]:

1. The state vector and covariance of state estimation error are initialized as

$$
\begin{aligned}
& \hat{\boldsymbol{\beta}}_{0 \mid 0}=\mathbb{E}\left(\boldsymbol{\beta}_{0}\right), \\
& \boldsymbol{P}_{0 \mid 0}=\mathbb{E}\left[\left(\boldsymbol{\beta}_{0}-\hat{\boldsymbol{\beta}}_{0 \mid 0}\right)\left(\boldsymbol{\beta}_{0}-\hat{\boldsymbol{\beta}}_{0 \mid 0}\right)^{T}\right],
\end{aligned}
$$

where, $\hat{\boldsymbol{\beta}}_{l \mid l-1}$ and $\hat{\boldsymbol{\beta}}_{l \mid l}$ represent the state estimates at the $l$ th step given the fringe sample observations up to and including the $(l-1)$ th and $l$ th pixel location, respectively.

2. The process updates of state vector and covariance matrix need not be performed since $\boldsymbol{F}$ is an identity matrix. Thus we set,

$$
\begin{aligned}
& \hat{\boldsymbol{\beta}}_{l \mid l-1}=\hat{\boldsymbol{\beta}}_{l-1 \mid l-1}, \\
& \boldsymbol{P}_{l \mid l-1}=\boldsymbol{P}_{l-1 \mid l-1} .
\end{aligned}
$$

3. The nonlinear observation vector model $\boldsymbol{h}_{l}\left(\boldsymbol{\beta}_{l}\right)$ can be linearized around the process updated state estimate $\hat{\boldsymbol{\beta}}_{l \mid l-1}$ using the first order Taylor series expansion as

$$
\boldsymbol{h}_{l}\left(\boldsymbol{\beta}_{l}\right)=\boldsymbol{h}_{l}\left(\hat{\boldsymbol{\beta}}_{l \mid l-1}\right)+\left.\frac{\partial \boldsymbol{h}_{l}}{\partial \boldsymbol{\beta}}\right|_{\hat{\boldsymbol{\beta}}_{l \mid l-1}}\left(\boldsymbol{\beta}_{l}-\hat{\boldsymbol{\beta}}_{l \mid l-1}\right),
$$

where the partial derivative matrix $\boldsymbol{H}_{l}=\left.\frac{\partial \boldsymbol{h}_{l}}{\partial \boldsymbol{\beta}}\right|_{\hat{\boldsymbol{\beta}}_{l \mid l-1}}$ is computed as $\boldsymbol{H}_{l}=-\hat{b}\left[\begin{array}{l}\sin \left(\hat{\phi}_{1}\right), \sin \left(\hat{\phi}_{1}\right) \bar{x}, \sin \left(\hat{\phi}_{1}\right) \bar{y}, \sin \left(\hat{\phi}_{1}\right) \bar{x}^{2}, \sin \left(\hat{\phi}_{1}\right) \overline{x y}, \sin \left(\hat{\phi}_{1}\right) \bar{y}^{2}, 0,-\cos \left(\hat{\phi}_{1}\right) / \hat{b} \\ 0, \sin \left(\hat{\phi}_{2}\right) \bar{x}, \sin \left(\hat{\phi}_{2}\right) \bar{y}, \sin \left(\hat{\phi}_{2}\right) \bar{x}^{2}, \sin \left(\hat{\phi}_{2}\right) \overline{x y}, \sin \left(\hat{\phi}_{2}\right) \bar{y}^{2}, \sin \left(\hat{\phi}_{2}\right),-\cos \left(\hat{\phi}_{2}\right) / \hat{b}\end{array}\right]$

The phase estimates $\hat{\phi}_{1}$ and $\hat{\phi}_{2}$ are obtained by substituting the estimated phase parameters $\left(\hat{\boldsymbol{\beta}}_{l \mid l-1}\right)$ in Eqs. (7) and (8), respectively. 
4. Based on the partial derivative matrix $\boldsymbol{H}_{l}$, the observation update of the state vector is performed as

$$
\begin{aligned}
\boldsymbol{K}_{l} & =\boldsymbol{P}_{l \mid l-1} \boldsymbol{H}_{l}^{T}\left(\boldsymbol{H}_{l} \boldsymbol{P}_{l \mid l-1} \boldsymbol{H}_{l}^{T}+\boldsymbol{R}_{l}\right)^{-1}, \\
\hat{\boldsymbol{\beta}}_{l \mid l} & =\hat{\boldsymbol{\beta}}_{l \mid l-1}+\boldsymbol{K}_{l}\left[\boldsymbol{I}_{l}-\boldsymbol{h}_{l}\left(\hat{\boldsymbol{\beta}}_{l \mid l-1}\right)\right], \\
\boldsymbol{P}_{l \mid l} & =\left(\boldsymbol{I}-\boldsymbol{K}_{l} \boldsymbol{H}_{l}\right) \boldsymbol{P}_{l \mid l-1}\left(\boldsymbol{I}-\boldsymbol{K}_{l} \boldsymbol{H}_{l}\right)^{T}+\boldsymbol{K}_{l} \boldsymbol{R}_{l} \boldsymbol{K}_{l}^{T},
\end{aligned}
$$

where, $I$ represents the identity matrix of size $2 \times 2, R_{l}$ is the observation noise covariance matrix and $\boldsymbol{K}_{l}$ is the EKF gain.

5. Once all the fringe samples are exhausted, the estimate of phase step is computed as

$$
\hat{\alpha}=\hat{\boldsymbol{\beta}}(7)-\hat{\boldsymbol{\beta}}(1) .
$$

In order to improve the robustness of phase step estimation, the phase parameter estimation procedure described above is performed at the immediate surrounding pixels of the selected location. Median value of the phase step estimates is chosen so that the contribution due to the outlier estimate is neglected. Utilizing the estimated phase step, the interferogram demodulation for the phase estimation is performed as

$$
\hat{\boldsymbol{\phi}}(x, y)=\arctan \left[\frac{\tilde{\boldsymbol{I}}_{1}(x, y) \cos (\hat{\alpha})-\tilde{\boldsymbol{I}}_{2}(x, y)}{\tilde{\boldsymbol{I}}_{1}(x, y) \sin (\hat{\alpha})}\right]
$$

\section{Simulation and Experimental results}

We provide a simulation example to demonstrate the ability of the proposed method for the estimation of two dimensional quadratic phase parameters. Two-dimensional signals of size $41 \times 41$ were simulated at signal to noise ratio (SNR) $=20 \mathrm{~dB}$ in function of phase step $\alpha$ with the quadratic phase parameters, $c_{0}=0, c_{1}=6 \mathrm{e}-2, c_{2}=5 \mathrm{e}-2$, $c_{3}=2 \mathrm{e}-4, c_{4}=3 \mathrm{e}-4, c_{5}=1 \mathrm{e}-4, c_{6}=c_{0}+\alpha$ and amplitude, $b=2$. The following intialization parameters are utilized in all of the simulation and experimental results

${ }_{65}$ provided in this paper. 


\begin{tabular}{|c|c|}
\hline$L$ & 15 \\
\hline$\hat{\boldsymbol{\beta}}_{0 \mid 0}$ & {$[0,1 \mathrm{e}-1,1 \mathrm{e}-1,1 \mathrm{e}-3,1 \mathrm{e}-3,1 \mathrm{e}-3,0, \hat{b}], \hat{b}=0.5 \cdot\left[\max \left(\tilde{I}_{1 L}\right)-\min \left(\tilde{I}_{2 L}\right)\right]$} \\
\hline $\boldsymbol{P}_{0 \mid 0}$ & $\operatorname{diag}([1 \mathrm{e} 2,1 \mathrm{e} 1,1 \mathrm{e} 1,1 \mathrm{e}-1,1 \mathrm{e}-1,1 \mathrm{e}-1,1 \mathrm{e} 2,1 \mathrm{e} 1])$ \\
\hline $\boldsymbol{R}_{l}$ & $1 \mathrm{e} 1 \cdot \boldsymbol{I}$ \\
\hline
\end{tabular}

where, $\operatorname{diag}(\cdot)$ is a function which forms a diagonal matrix with a diagonal vector provided as an argument.
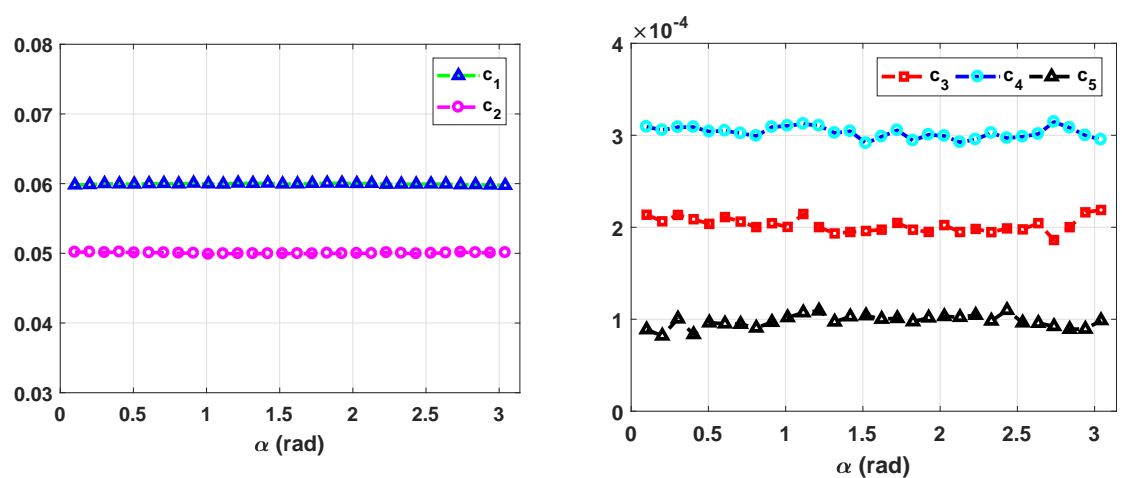

Figure 1: Estimates of (a) $c_{1}, c_{2}$ and (b) $c_{3}, c_{4}, c_{5}$ in function of $\alpha$. The simulation was performed with two-dimensional signals $\left(I_{1}\right.$ and $\left.I_{2}\right)$ of size $41 \times 41$.

Figure 11 shows the estimated quadratic phase parameters plotted in function of $\alpha$. It can be observed that the proposed method is capable of accurately estimating the phase parameters for a wide range of phase step values. A simulation example is provided for the demodulation of interferograms shown in Fig. 2, The interferograms were simulated with the phase step of $\alpha=1 \mathrm{rad}$ at $\mathrm{SNR}=20 \mathrm{~dB}$. For each value of $\alpha$, mean estimate of 50 simulation run is considered. The fringe amplitude was set as $b(x, y)=25 \cdot \exp \left\{-\left[\left(x^{2}+y^{2}\right) / 5 \mathrm{e} 4\right]\right\}$. In the implementation of the proposed method, the pixel location with coordinates $x_{p t}=126$ and $y_{p t}=123$ was selected to evaluate the phase step. A red colored rectangle surrounding the selected pixel is shown in Fig. 2b. The same pixel location is considered in all the simulation and experimental results provided in this paper. Figure 3 shows the estimated phase maps computed 


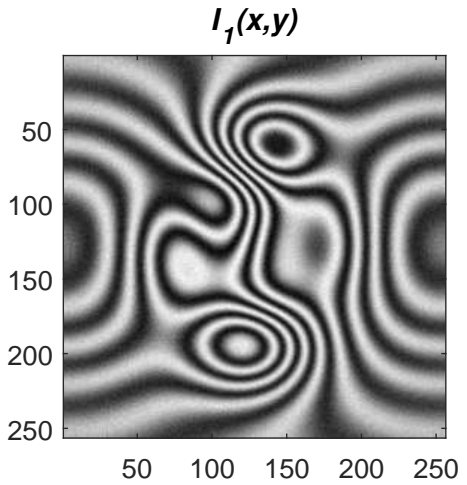

(a)

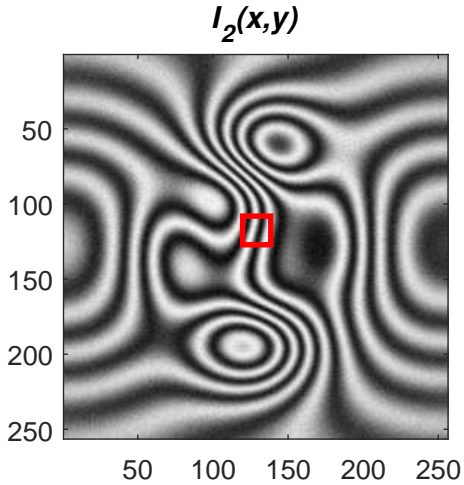

(b)

Figure 2: Phase shifted interferograms of size $256 \times 256$ simulated with $\alpha=1$ at $\mathrm{SNR}=20 \mathrm{~dB}$. Fig. 2b shows a red colored rectangle sourrounding the selected pixel $\left(x_{p t}=126, y_{p t}=123\right)$ for the phase-step estimation.

using the Kries, ST, OF, GS, EVI and the proposed method. Whereas the Kries, ST and OF methods failed to provide reliable phase estimates, the GS, EVI and the proposed method resulted in accurate phase estimates. For the same simulation example, the phase estimation was performed in function of $\alpha$. Figures $4 \mathrm{a}$ and $4 \mathrm{~b}$ show plots of root mean square errors (rmse) in the phase estimates computed at $\mathrm{SNR}=\infty \mathrm{dB}$ and SNR $=20 \mathrm{~dB}$, respectively. The GS, EVI and the proposed methods are found to be capable of accurate phase estimation for a wide range of $\alpha$ values. However, since the proposed method is developed with the main objective of accurate phase step estimation, it is more appropriate to evaluate the performance of the proposed method for the estimation of phase step instead of phase. Accordingly, errors in phase step evaluation using the EVI and the proposed method are compared in Fig. $4 \mathrm{c}$ at $\mathrm{SNR}=10 \mathrm{~dB}$ and $30 \mathrm{~dB}$. The proposed method is found to be more noise tolerant compared to the EVI method over the entire range of $\alpha$. A study is also performed to observe the errors in the phase step estimate computed at five distinct pixel locations $\left(x_{p t}, y_{p t}\right)=\{(106,122),(46,132),(63,202),(120,195),(226,125)\}$. The errors are plotted in Fig. 4d Note that these locations were selected at random. The error values indicate that the phase step estimation is mostly insensitive to the selection of pixel locations. 


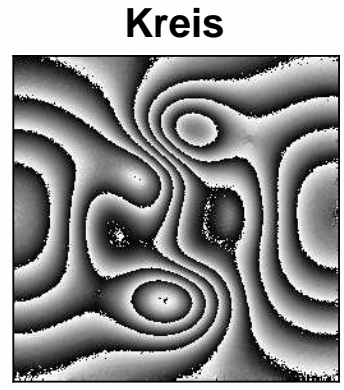

(a)

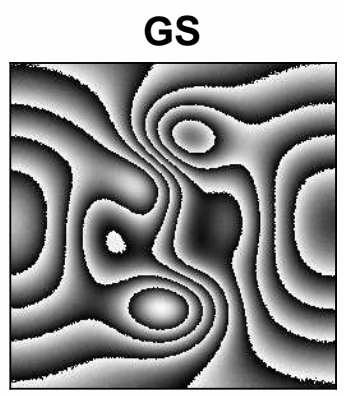

(d)

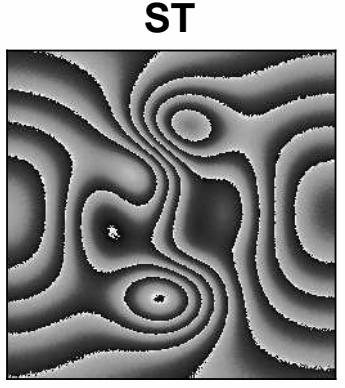

(b)

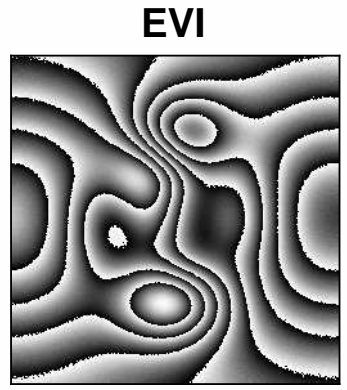

(e)

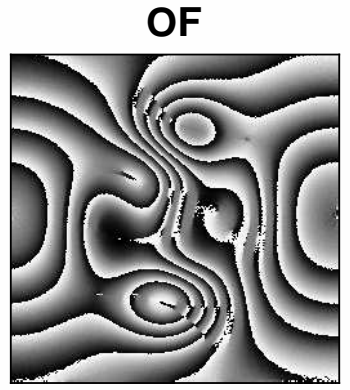

(c)

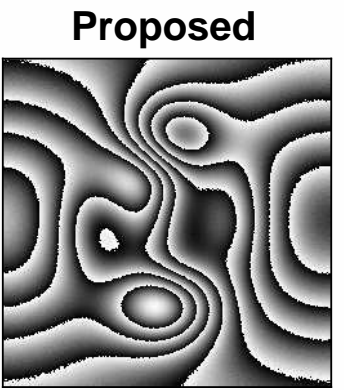

(f)

Figure 3: Estimated phase maps from the phase shifted interferograms shown in Fig. 2 using (a) Kries (b) ST (c) OF (d) GS (e) EVI and (f) the proposed method.

Figure 5 shows the results of phase step estimation performed at three different pixel locations in the interferogram given in Figure 2 a). The interferograms were simulated at $\mathrm{SNR}=10 \mathrm{~dB}$ with $\alpha=0.25 \mathrm{rad}$. The second and third columns show the reconstructed wrapped phase and fringe patterns, respectively, evaluated using the estimated polynomial coefficients. The results indicate that the proposed method is capable of reliable estimation of the phase step at any user selected pixel. A study of the effect of window size on the accuracy of phase step estimation is performed as shown in Figure 6 The results suggest that window sizes from the set $L=[15,19]$ provide good accuracy in phase step estimation over a range of signal to noise ratio. The proposed method was implemented using MATLAB R2017a on 3.19-GHz Intel core i5 processor machine with $8-\mathrm{GB}$ random access memory. The computation time 


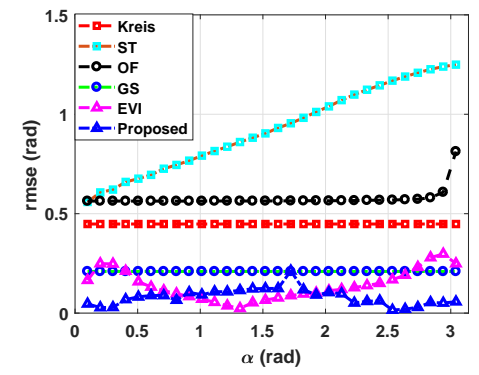

(a)

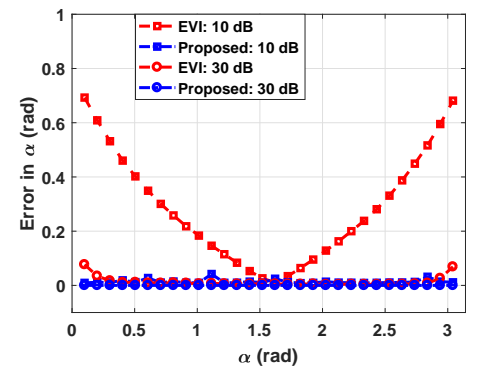

(c)

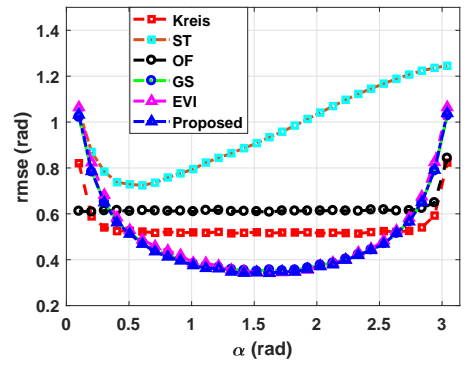

(b)

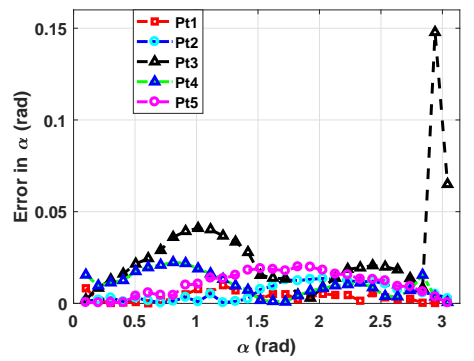

(d)

Figure 4: Root mean square error in the estimation of phase computed using the Kries, ST, OF, GS, EVI and the proposed method in function of $\alpha$ at (a) SNR $=\infty \mathrm{dB}$ and (b) $\mathrm{SNR}=20 \mathrm{~dB}$. (c) Error in the estimation of phase step computed using the EVI and the proposed method in function of $\alpha$ at SNR $=10$ and $30 \mathrm{~dB}$. (d) Error in the estimation of phase step computed using the proposed method in function of $\alpha$ at different pixel locations (represented as Pt\#).

required by the proposed method with $L=17$ was found to be around 0.3 seconds. Note that this computation time corresponds to the phase step estimation performed total nine pixel locations at and around the user selected pixel.

The experimental validation of the proposed method is performed with the interferograms recorded in a classical phase shifting holographic interferometry setup. Two of five frames shown in Figs. 7 $\mathrm{b}$ and $7 \mathrm{~b}$ correspond to the out-of-plane displacement of an aluminum plate. The Schwider-Hariharan phase shifting algorithm [25, 26] was implemented with the five phase-shifted frames to obtain the reference phase map shown in Fig. 77. The demodulated phase maps computed using the Kries, ST, OF, GS, EVI and the proposed method are shown in Fig. 8 Similar to the simulation results, the estimated phase maps obtained using the GS, EVI and the proposed method are found 

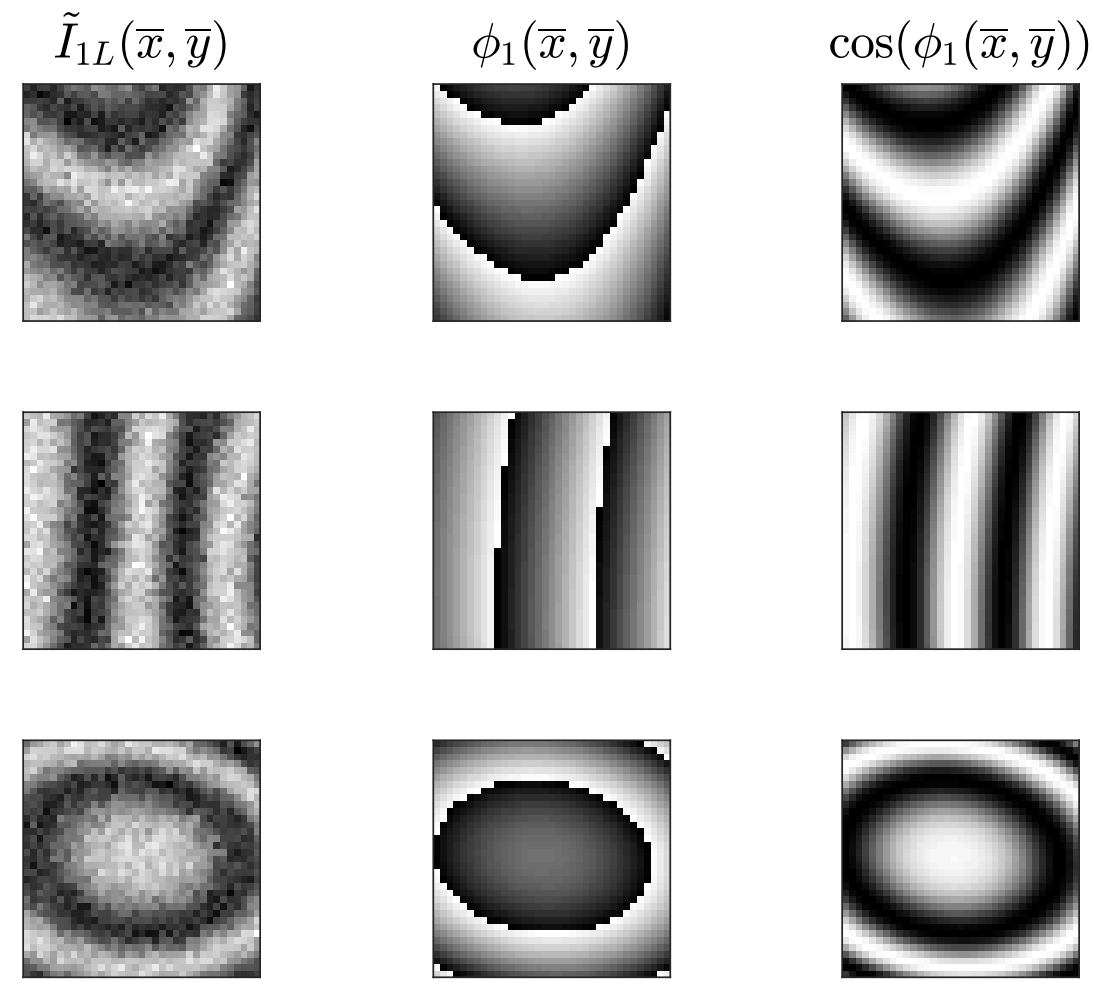

Figure 5: The first, second and third column in the figure show original windowed interferograms, the reconstructed wrapped phase and fringe patterns at (a) $x_{p t}=106, y_{p t}=122$ (b) $x_{p t}=46, y_{p t}=132$ and (c) $x_{p t}=120, y_{p t}=202$, respectively.

to be more reliable in comparison with those obtained using the Kries, ST and OF methods.

\section{Discussion}

The method proposed in [21] considers the Zernike polynomial phase fitting within a locally masked interferogram. The phase shift is derived from the estimated coefficients of the polynomials. Whereas the number of polynomial coefficients required to be estimated in [21] are eleven, only eight coefficients need to be estimated in the proposed method. Since the coefficient estimation is a nonlinear optimization process, the 

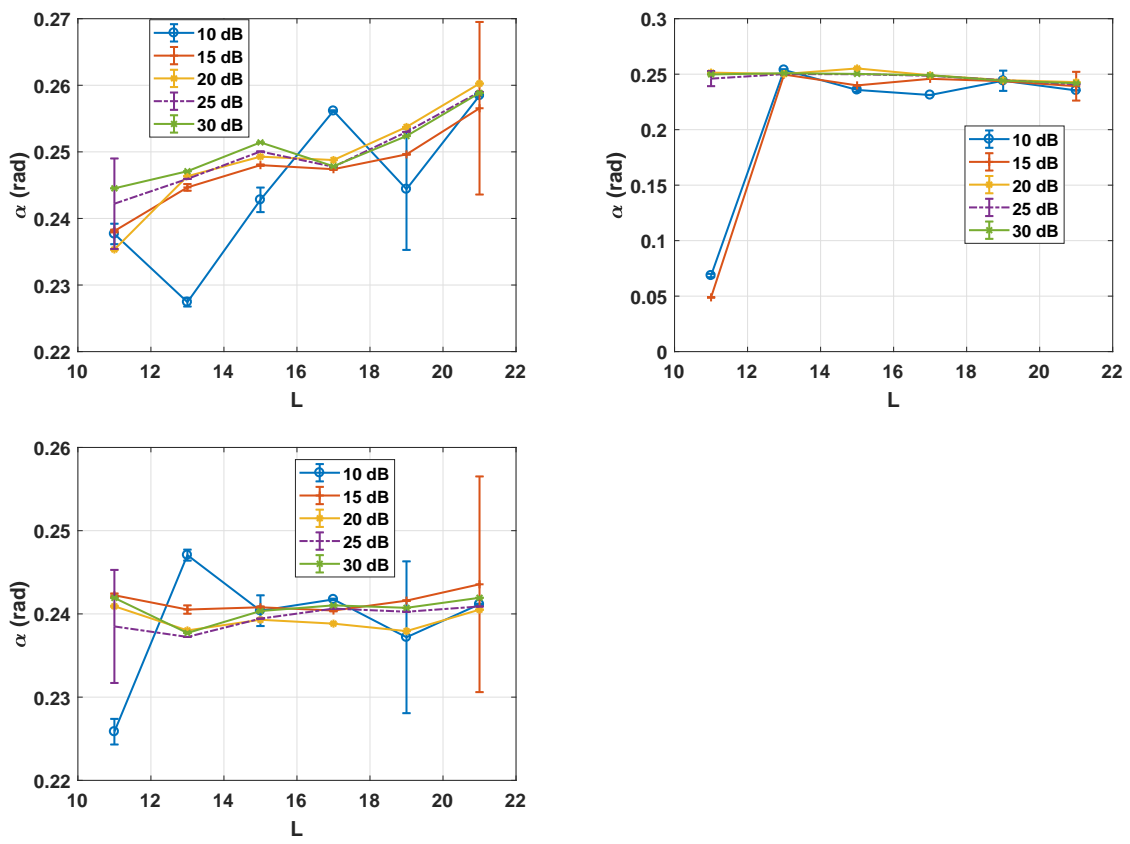

Figure 6: Estimated phase step ( $\alpha=0.25 \mathrm{rad}$ ) at (a) $x_{p t}=106, y_{p t}=122$ (b) $x_{p t}=46, y_{p t}=132$ and (c) $x_{p t}=120, y_{p t}=202$ in function of window size $L$ and SNR.

computational complexity increases with the increase in the number of coefficients. In order to have a reliable approximation of phase with a small number of polynomial coefficients, the size of the local mask needs to be small. However, in this case, the noise robustness of the optimization algorithm tends to decrease. Since the proposed method utilizes Kalman filtering which is capable of handling noise in a very effective manner, the required local mask size is smaller compared to the method in [21]. It is important to note that whereas the coefficient set in [21] does not include the fringe amplitude, the fringe amplitude in the proposed method is estimated as a part of the process and has been considered to be spatially varying in the simulation and experimental studies.

Since the quadratic phase approximation is considered only within small size window around the user selected pixel, the proposed method is applicable for the analysis of interferograms with widely varying fringe densities including those containing inherent phase discontinuities. However, it is important to note that the pixel should be selected such that the analysis window does not contain pixels from the phase discon- 


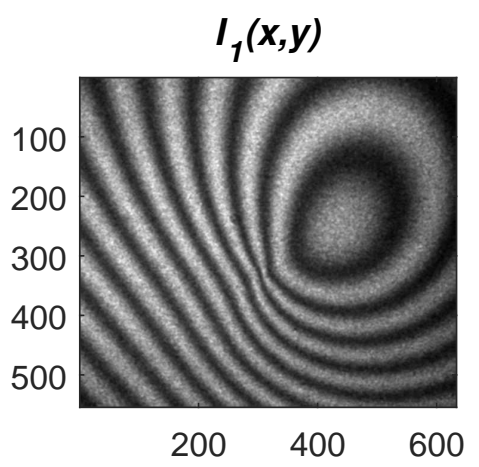

(a)

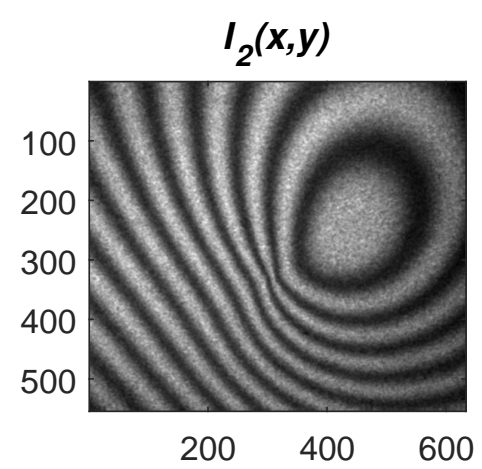

(b)

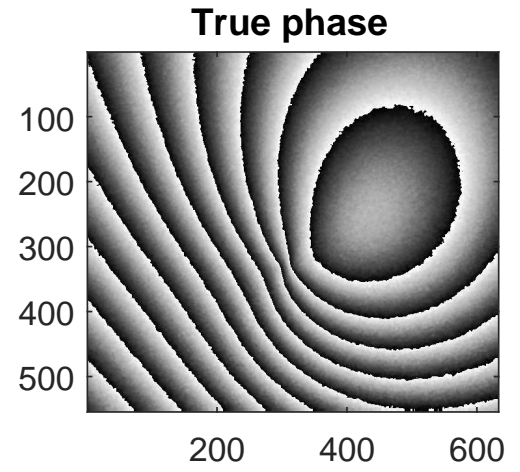

(c)

Figure 7: (a) and (b) Two phase-shifted interferograms recorded in a classical holographic interferometric setup corresponding to out-of-plane deformation of an aluminum plate. (c) The reference true phase computed using the Schwider-Hariharan (5-step) phase shifting algorithm.

tinuous region.

\section{Conclusion}

In the proposed method, a quadratic phase parameter estimation method based on the state space analysis is found to provide a robust estimate of phase step in a two-step PSI system. The utilization of spatial information on interferograms and appropriate assumption on the local phase profile allows the method to effectively handle the presence of noise. Phase parameter estimates obtained using the EKF based state space analysis are found to converge towards their true values without any prior knowledge 


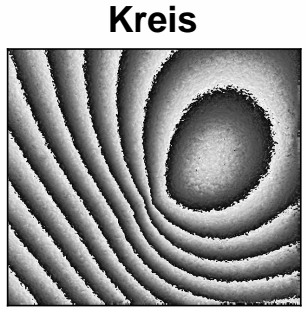

(a)

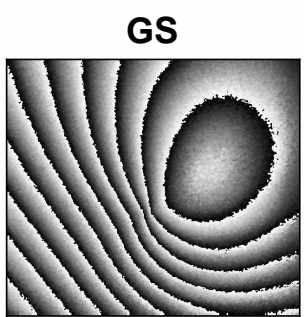

(d)

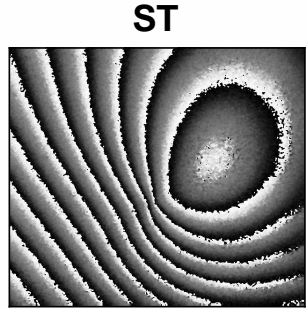

(b)

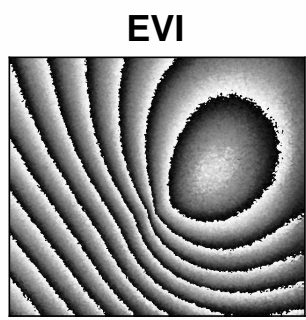

(e)

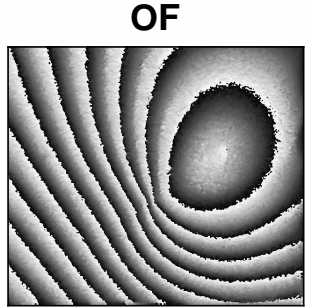

(c)

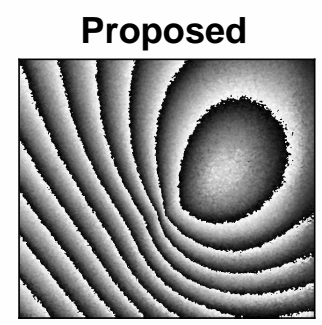

(f)

Figure 8: Estimated phase maps from the phase shifted interferograms shown in Fig. 7 using (a) Kries (b) ST (c) OF (d) GS (e) EVI and (f) the proposed method.

about them. This is justified with the use of same initial conditions in all the simulation and experimental results. A uniform performance is observed over a wide range of phase step and SNR values. The comparison of numerical and experimental performances with previously suggested methods demonstrates the practical applicability of the proposed method.

\section{References}

[1] Creath K. Phase-shifting speckle interferometry. App Opt 1985;24:3053-3058.

[2] Patil A, Rastogi P. Moving ahead with phase. Opt Lasers Eng 2007;45:253-257.

[3] Servin M, Quiroga J A, Padilla M Fringe Pattern Analysis for Optical Metrology: Theory, Algorithms, and Applications. Wiley-VCH; 2014. 
[4] Kulkarni R, Rastogi P. Phase estimation: temporal fringe analysis. In: Single and multicomponent digital optical signal analysis, IOP Publishing; 2017, p.8-1 to $8-31$.

[13] Luo C, Zhong L, Sun P, Wang H, Tian J, Lu X. Two-step demodulation algorithm based on the orthogonality of diamond diagonal vectors. Appl Phys B 2015;119(2):387-391. 
[23] Quiroga J A, Servin M. Isotropic n-dimensional fringe pattern normalization. Opt Comm 2003;224:221-227.

[24] Simon D. Optimal State Estimation: Kalman, H Infinity, and Nonlinear Approaches. Wiley-Interscience; 2006. based on the quotient of inner products of phase-shifting interferograms. J Opt 2015;17(8):085703.

[15] Maciek W, Zofia S, Krzysztof P. Two-frame tilt-shift error estimation and phase demodulation algorithm. Opt Lett 2015;40:3460-3463.

[16] Rivera M, Dalmau O, Gonzalez A, Hernandez-Lopez F. Two-step fringe pattern analysis with a Gabor filter bank. Opt Lasers Eng 2016;85:29-37.

[17] Xu X, Shou J, Lu X, Yin Z, Tian J, Li D, Zhong L. Independent component analysis based two-step phase retrieval algorithm. J Opt 2016;18:105701(7pp).

[18] Chao T, Shengchun L. Two-frame phase-shifting interferometry for testing optical surfaces. Opt Exp 2016;24:18695-18708.

[19] Chao T, Shengchun L. Demodulation of two-shot fringe patterns with random phase shifts by use of orthogonal polynomials and global optimization. Opt Exp 2016;24:3202-3215.

[20] Dalmau O, Rivera M, Gonzalez A. Phase shift estimation in interferograms with unknown phase step. Opt Comm 2016;372:37-43.

[21] Chao T, Shengchun L. Phase retrieval in two-shot phase-shifting interferometry based on phase shift estimation in a local mask. Opt Exp 2017;25:21673-21683.

[22] Dominika S, Maciej T, Krzysztof P. Evaluation of adaptively enhanced twoshot fringe pattern phase and amplitude demodulation methods. Appl Opt 2017;56:5489-5500.

[14] Niu W, Zhong L, Sun P, Zhang W, Lu X. Two-step phase retrieval algorithm 
[25] Schwider J, Burow R, Elssner K E, Grzanna J, Spolaczyk R, Merkel K. Digital wave-front measuring interferometry: some systematic error sources. Appl Opt $1983 ; 22: 3421-3432$.

[26] Hariharan P, Oreb B F, Eiju T. Digital phase-shifting interferometry: a simple error-compensating phase calculation algorithm. Appl Opt 1987;26:2504-2506. 